\title{
Redécouverte et Écologie d'Onopordum algeriense (Munby) Pomel = Carduus algeriensis Munby, endémique stricte en voie d'extinction du secteur algérois
}

\author{
Selma Amina Djelid ${ }^{(1,2,3)}$, Teresa Calvão ${ }^{(2,3)}$, Sahraoui Bensaid ${ }^{(1)}$ \\ ${ }^{1}$ Laboratoire d'Ecologie végétale, Faculté des Sciences Biologiques, Université des Sciences et de la Technologie Houari \\ Boumediène, BP 32 El Alia Alger 16111 Algerie. \\ ${ }^{2}$ Department of Sciences and Environmental Engineering, NOVA School of Science and Technology, NOVA \\ University Lisbon, Campus de Caparica, 2829-516 Caparica, Portugal \\ ${ }^{3}$ Center for Environmental and Sustainability Research (CENSE), NOVA School of Science and Technology, NOVA \\ University Lisbon, Campus de Caparica. 2829-516 Caparica, Portugal
}

Correspondencia: Selma Djelid e-mail: djelidaminaselma@yahoo.fr Recibido: 15 marzo 2019

Aceptado: 9 septiembre 2020

Publicado on-line: 24 noviembre 2020

Editado por: Andrés V. Pérez Latorre

\section{Résumé}

Onopordum algeriense est une espèce endémique stricte du secteur algérois, limitée plus précisément aux collines et montagnes du Puits des Zouaves (Forêt de Baïnem). Compte tenu de sa distribution très restreinte on comprend I'importance de sa conservation. Elle est effectivement strictement protégée en Algérie par Décret Exécutif.

L'objectif de cet article consiste dans l'évaluation de la dimension de la population et du statut de conservation d'Onopordum algeriense selon les critères de l'UICN ainsi que sa vulnérabilité. Un échantillonnage exhaustif par la méthode des quadrats a été réalisé dans deux sites dans la forêt de Baïnem.

Afin d'étudier la distribution spatiale de l'espèce on a appliqué la méthode des Polygones de Thiessen et l'Indice de Moran suivie d'une analyse canonique de corrélation pour élucider la relation entre les variables du milieu physique et la répartition des individus de l'espèce. Les résultats ont révélé une tendance à l'agrégation de l'espèce.

La dimension de la population est réduite (on a recensé 1648 pieds sur $320 \mathrm{~m}^{2}$ ). Onopordum algeriense semble perturbé par la présence du sanglier et probablement aussi par la présence d'un coléoptère (la cétoine funeste). Les conditions particulières de son habitat naturel ne semblent pas indispensables à sa survie mais l'absence de compétiteurs dans cet habitat représentera, sans doute, un fort avantage.Selon les critères de I'UICN adoptées, l'espèce est en danger critique. S'il n'y a pas un effort de conservation I' avenir de l'espèce semble incertain aussi du fait de la pression pour l'urbanisation dans cette zone.

Mots clés: Carduus algeriensis, Onopordon algeriense, endémisme, rare, extinction, Algérie, UICN.

\begin{abstract}
Rediscovery and Ecology of Onopordum algeriense (Munby )Pomel = Carduus algeriensis Munby, a strict endemic species in the process of extinction of the Algerian sector. Onopordum algeriense is a strict endemic species of the Algerian sector, limited more precisely to the hills and mountains of the Puits des Zouaves (Baïnem Forest). Given its very restricted distribution its conservation is essential. It is indeed strictly protected in Algeria by Executive Decree.

The objective of this paper is to assess the population size and conservation status of Onopordum algeriense according to IUCN criteria as well as its vulnerability. Exhaustive sampling by the quadrat method was carried out in two sites in the Baïnem forest.

In order to study the spatial distribution of the species, the method of Thiessen Polygons and Moran Index followed by a canonical correlation analysis was applied to elucidate the relationship between the environment variables and the spatial distribution of the species. The results revealed a trend towards aggregation. The population has a reduce size (1648 feet by $\left.320 \mathrm{~m}^{2}\right)$. Onopordum algeriense seems to be disturbed by the presence of wild boar and probably also by the presence of a beetle (the evil ketone). The particular conditions of its natural habitat do not seem indispensable to its survival but the absence of competitors in this habitat will, no doubt, represent a strong advantage. According to the adopted IUCN criteria, the species is Critically Endangered. If there is not conservation effort the future of the species seems uncertain also because of the pressure for urbanization in this area.
\end{abstract}

Keywords: Carduus algeriensis, Onopordon algeriense, endemism, rare, extinction, Algeria, UICN. 


\section{Introduction}

Le bassin méditerranéen est le troisième hot spot le plus riche du monde en diversité végétale (Médail $\&$ al. 2004), cette richesse est en rapport avec des facteurs historiques et écologiques (Petit \& al., 2002; Quezel, 2003). L'ensemble de l'Afrique du Nord, majoritairement représenté par l'Algérie, est donc au cœur des préoccupations mondiales en matière de biologie de la conservation: richesse taxonomique avérée, fort endémisme végétal et menaces anthropiques croissantes (Vela \& Benhouhou, 2007).

Onopordum algeriense (Munby) Pomel (Synonyme: Carduus algeriensis Munby) est une espèce endémique à distribution restreinte: Région méditerranéenne, Sous-région eu méditerranéenne, Domaine maghrébin méditerranéen, Secteur algérois, Soussecteur littoral.

Compte tenu de sa distribution très restreinte on comprend l'importance de sa conservation.

Onopordum algeriense est une plante de grande taille (10-180 cm de haut) à nombreuses tiges fines, ramifiées, annuelle ou bisannuelle. II s'agit d'une plante héliophile colonisant des milieux ouverts, ensoleillés et les éboulis.

Cette espèce a été trouvée la première fois par Munby en 1855, en Algérie. A peu près 100 ans plus tard elle est citée dans un article de I'Union Internationale pour la Conservation de la Nature et de ces Ressources (Faurel, 1958). Elle a été retrouvée par nos soins lors d'une herborisation en 2012 aux Puits des Zouaves, Forêt de Baïnem, mais seulement en 2016 fut possible un inventaire plus détaillé sur le terrain.

La Forêt de Baïnem a un statut domaniale publique et géré par la Direction Générale des Forêts - ministère de l'agriculture -Algérie. Tout autour, et même á l'intérieur de la forêt, il y des urbanisations qui conduisent à des pressions sur les communautés végétales, comme sol remué, déchets de construction, piétinement.

La détermination des espèces de ce genre se heurtent souvent à une grande difficulté $d^{\prime}$ 'identification, liée à la variabilité des taxons. Ce qui peut expliquer cette lacune d'à peu près un siècle.

L'objectif de cet article est de donner à connaitre la distribution de l'espèce et de mettre en évidence le statut actuel d'une espèce endémique à distribution restreinte, en Algérie, selon les critères de I'UICN.

\section{Matériels et Méthodes}

\section{La zone d'étude}

Située sur la côte algéroise, la forêt de Baïnem se trouve à $15 \mathrm{~km}$ à l'ouest d'Alger centre, et s'étend sur 508 ha (Fig. 1. A), entre 80 et 302 m d'altitude, dans un terrain très accidenté au versant Nord, entrecoupé de ravins encaissés donnant naissance à quelques oueds. Au centre, la topographie est plus adoucie, là sont effectués des aménagements réactifs, et l'installation des parcelles expérimentales. Quant au versant Sud, il est en général de pente moyenne à faible, mais aussi avec des pentes supérieures à $50 \%$ (rudes), et moyennement en exposition Est et Ouest, donnant un relief rectiligne. Le bioclimat est subhumide à hiver chaud. Le terrain est diversifié avec la présence de roches métamorphiques (schistes, micaschistes, gneiss) (ISL-BRGM, 2006).

\section{Mesure de l'abondance d'Onopordum algeriense (Munby) Pomel}

On a mis en place un dispositif d'étude d'O. algeriense (Vela \& al., 2008 ; Baumel \& al., 2009 ; Youssef \& al., 2011). Après prospection de terrain, nous avons rencontré deux types de situations ou I'espèce apparait: répartition à forte pente (site 1) et répartition en terrain plat (site 2). Les deux sont éloignés à peu près $500 \mathrm{~m}$.

Dans le présent article on appelle « site » le lieu de présence d'O. algeriense. Comme la distribution de l'espèce à l'intérieur des sites n'est pas homogène, on a déployé diverses placettes au sein des sites. On appelle « placette » l'endroit exact où l'espèce sera étudiée. Un site peut donc contenir une ou plusieurs placettes distantes de quelques dizaines de mètres. Un quadrat (1metre* 1 metre) a été déplacé sur la placette en suivant un cheminement déterminé au préalable (Fig. 1. B). Dans le site 1 on a déployé 3 placettes. La placette 2 a été subdivisée en deux parties (Partie 1 et partie 2). Le site 2 englobe seulement 1 placette.

L'échantillonnage est de type exhaustif afin d'inventorier tous les individus dans chaque quadrat. Les coordonnées géographiques de chaque pied ont été déterminées à l'aide d'un système de positionnement globale GPS.

Pour chaque quadrat on a évalué des variables relatives à l'espèce (Système de Codage):

Le nombre d'individus, le recouvrement (pourcentage), la hauteur des pieds, la présence ou absence de capitules.

Et des variables relatives au milieu physique:

La pente (pourcentage), Présence ou absence de perturbation dans le quadrat, exprimée par un indice (traces sangliers, remblai, déchets, bouse de vache, piétinement, bord de route).

Cette méthode d'échantillonnage donne différents types d'information: 1) L'estimation de l'effectif d'O. algeriense dans la placette. 2) Des données sur l'intensité des facteurs de perturbation ou de compétition qui seront utilisés comme variables explicatives dans la cartographie de l'abondance de l'espèce, puisque tous les quadrats sont géo référenciées. Ces données permettent aussi l'application des techniques d'analyse spatiale: Polygones de Thiessen ou diagramme de Voronoï et Indice de Moran. 


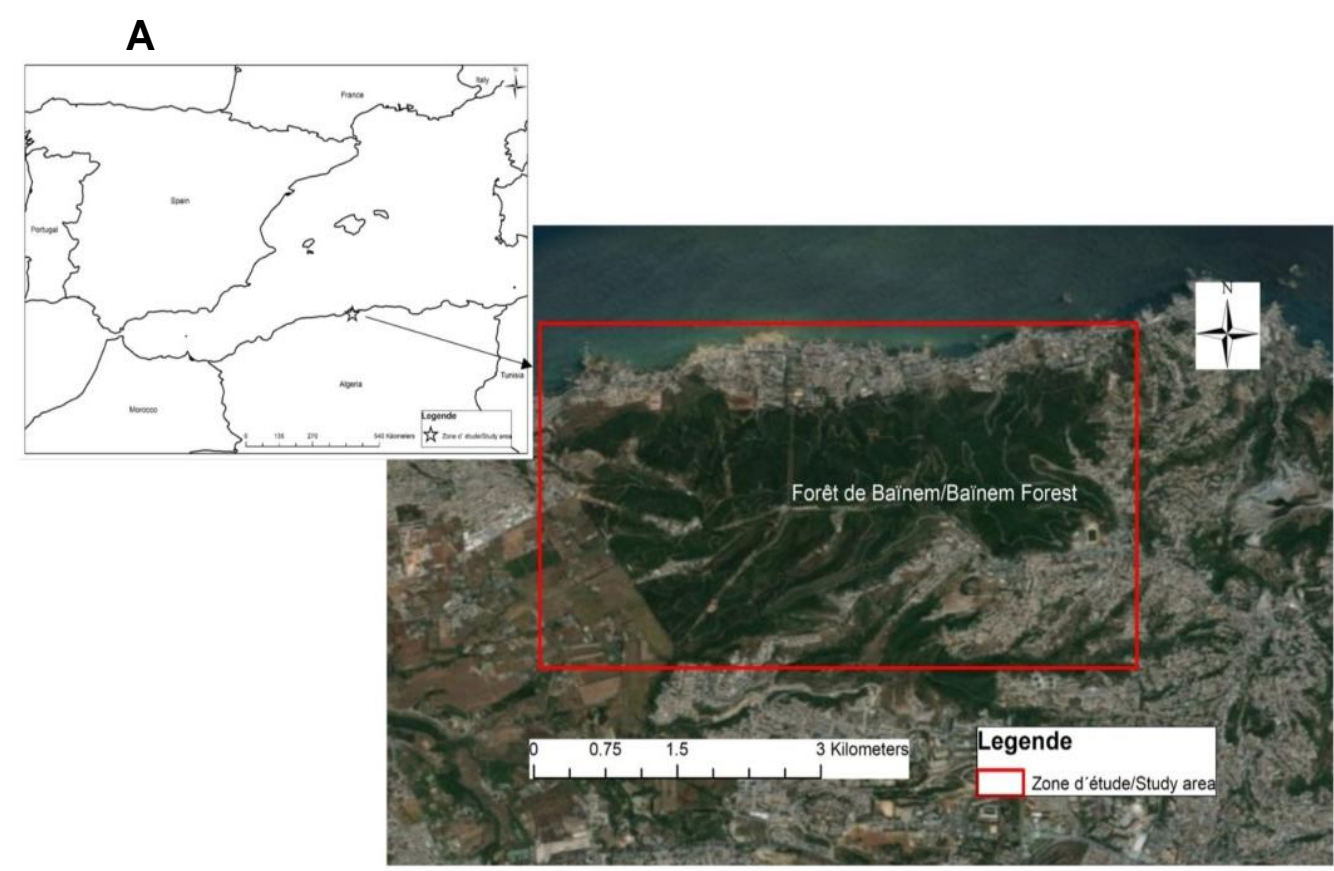

\section{B}

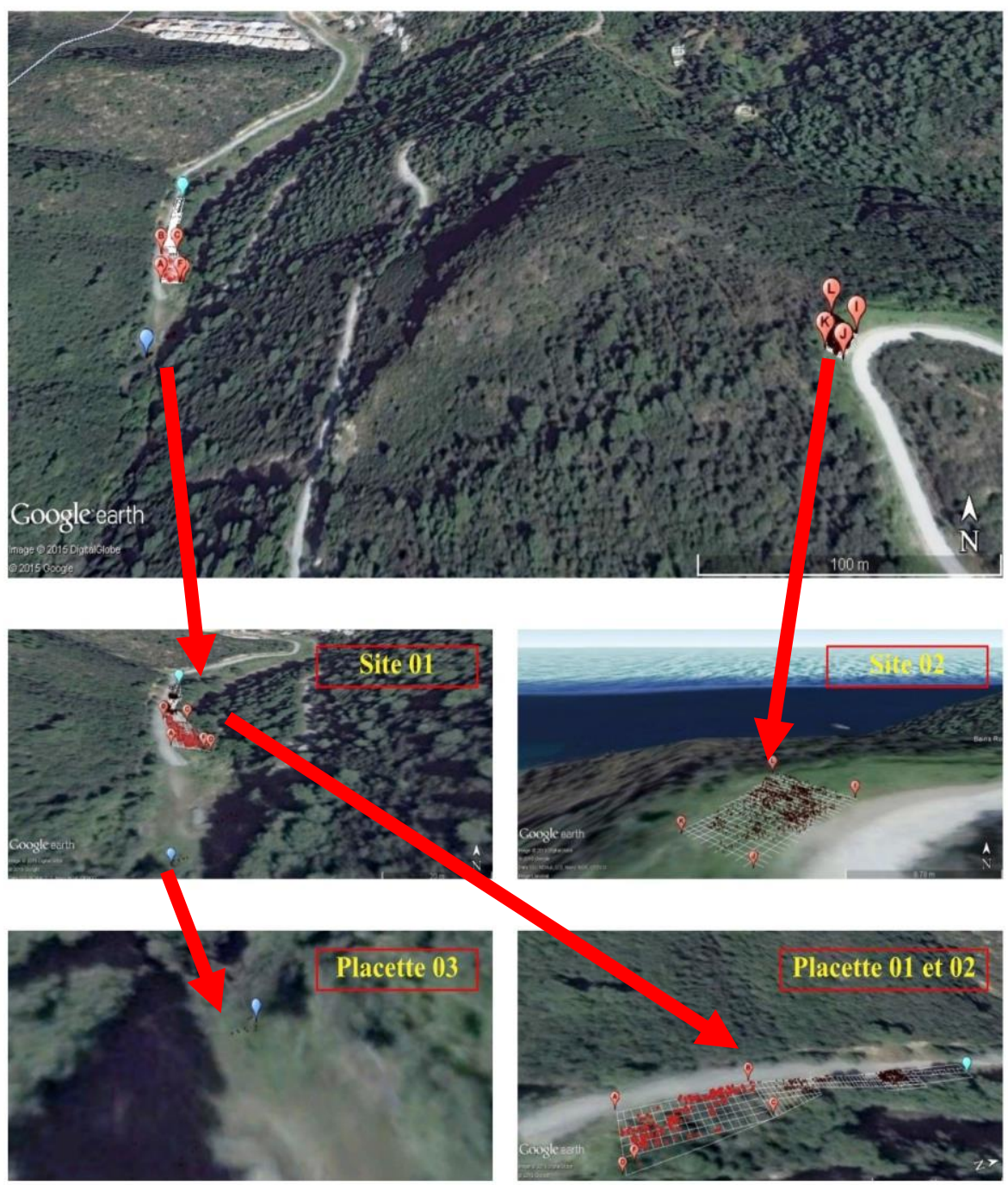

Figure 1. A. Localisation et délimitation de la zone d'étude (Image satellite, Google Earth V7.1.2.2041, 2015). B. Protocole expérimental et son emplacement.

Figure 1.A. Localization and delimitation of the study area (Satellite image, Google Earth V7.1.2.2041, 2015). B. Experimental Protocol and its location. 
Pour les polygones de Thiessen (Fortin \& Dale, 2005) nous avons utilisé une fonctionnalité d'ArcMap (ESRI) 10.3, à partir d'un fichier Excel contenant les coordonnées géographiques de chaque pied.

Afin de déterminer la présence d'autocorrélation spatiale on a appliqué le test statistique Indice de Moran (I) qui mesure essentiellement la ressemblance entre voisins (Fortin \& Dale, 2005). Pour la détermination de l'indice de Moran on a utilisé une fonctionnalité d'ArcGis, à partir d'un fichier Excel contenant les coordonnées géographiques de chaque pied. L'indice a été déterminé pour chaque placette/ partie et ensuite la moyenne a été calculée.

\section{Analyse canonique des corrélations}

L'analyse spatiale à travers le diagramme de Voronoï montrant une structure agrégative, l'une des questions fondamentales est la suivante: existe-t-il une relation entre les variables écologiques et la répartition d'O. algeriense? Afin d'affiner cette analyse et comprendre le processus de colonisation du milieu, une analyse canonique de corrélation (Zuur et al., 2007) a été réalisée utilisant les variables relatives à l'espèce et les variables relatives au milieu à l'aide de logiciel XLSTAT 2014.5.03.

\section{Résultats et discussion}

\section{Analyse de données spatiales}

On a détecté un nombre total de 1648 pieds de l'espèce dans l'ensemble des deux sites (674 pour le site 1 et 974 pour le site 2). On a un total de 4 diagrammes de polygones de Thiessen pour le site 1 (1 pour la placette 1, 2 pour la placette 2 et 1 pour la placette 3 ) et 1 diagramme pour le site 2 présentés dans les Figures $2 \mathrm{~A}$ et $\mathrm{B}$ montrent une distribution agrégative d'O. algeriense. L'Indice de Moran prend la valeur de 0,041 , ce qui indique une tendance de l'espèce à l'agrégation. Les deux techniques d'analyse spatiale ont permis de donner une idée sur le type de distribution d'O. algeriense: l'abondance des individus dans les deux sites se caractérise par une hétérogénéité locale à distribution concentrée, cette
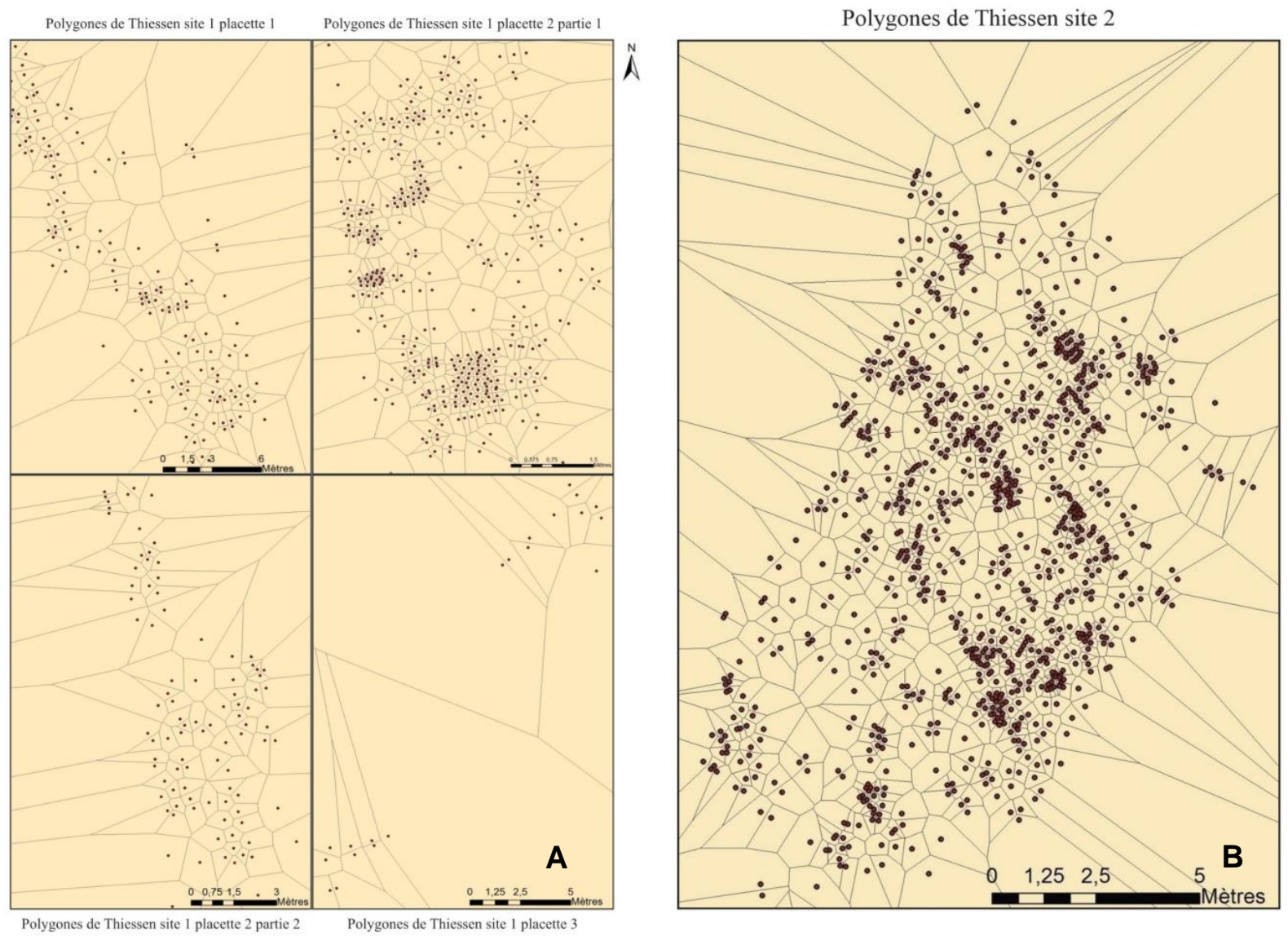

Figure 2.A: - Polygones de Thiessen site 01. B: - Polygones de Thiessen site 02.

Figure 2.A: - Polygons of Thiessen site 01. B: - Polygons of Thiessen site 02

hétérogénéité correspond à la variabilité entre les individus d'un même site.

\section{Analyse canonique des corrélations}

Dans la matrice des corrélations, en ce qui concerne les variables relatives à l'espèce, on note une forte corrélation entre le recouvrement et la hauteur des pieds $(0,541)$, entre le nombre d'indivi- dus et le recouvrement $(0,446)$, entre le nombre d'individus et la hauteur des pieds $(0,440)$ et entre le recouvrement et le nombre de capitules $(0,428)$. En ce qui concerne les variables relatives aux milieux physique, on remarque les suivantes corrélations: bord de route et piétinement $(0,830)$, piétinement et remblai $(-0,746)$, bord de route et remblais $(-0,616)$, 
traces sanglier et déchets $(0,483)$ et bord de route et pente $(0,327)$.

Le premier axe seul explique $67 \%$ de la variabilité des données (Fig. 3).

Les corrélations canoniques sur le facteur 1 montrent que les Coefficients de redondance $\mathrm{Y} 1$ et $\mathrm{Y} 2$ sont corrélées (Tableau 1).

Tableau 1: Coefficients de redondance $(Y 1)$ et $(Y 2)$.

Table 1: Redundancy coefficients $(Y 1)$ and $(Y 2)$.

\begin{tabular}{llll}
\hline \multicolumn{4}{l}{ Coefficients de redondance $(\mathrm{Y} 1)$} \\
\hline F1 & F2 & F3 & Somme \\
0,07 & 0,082 & 0,005 & 0,156 \\
\hline
\end{tabular}

\begin{tabular}{llll}
\multicolumn{4}{l}{ Coefficients de redondance $(Y 2)$} \\
\hline F1 & F2 & F3 & Somme \\
0,05 & 0,014 & 0,002 & 0,066
\end{tabular}

\begin{tabular}{lll}
\hline \multicolumn{3}{l}{ Différence entre coefficients } \\
\hline F1 & F2 & F3 \\
0,577 & 0,37 & 0,158 \\
\hline
\end{tabular}

On note que cette valeur est plus grande que les corrélations entre les facteurs.

Les coefficients de redondance 1 et 2 montrent qu'une faible portion de la variabilité des variables initiales est prédite par les variables canoniques.

Le recouvrement $(R G)$ et le nombre de capitules $(C)$ sont corrélés positivement avec le facteur 1. Ce qui explique que, plus le recouvrement est important, on a au moins un capitule par individu (Fig. 3).

On note aussi que le nombre d'individus d'O. algeriense (Nio) est corrélé négativement avec les perturbations - sanglier (IP2) sur le facteur 02. Cela veut dire que la présence du sanglier a un effet direct sur la prolifération d'O. algeriense.

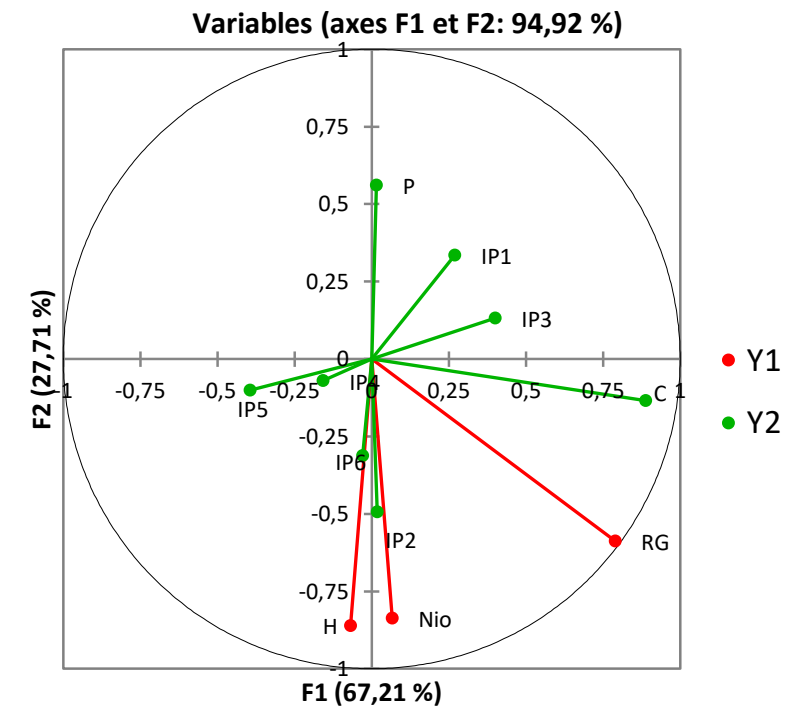

Figure 3. Cercle de corrélation canonique des variables des plans (1-2).

Figure 3. Circle of canonical correlation of the variables of the planes (1-2).

\section{Vulnérabilité et conservation d'Onopordum algeriense (Munby)Pomel:}

Afin de percevoir les facteurs affectant la distribution d'O. algeriense, une synthèse des phéno- mènes limitant sa propagation a été réalisée, avec information annotée sur le terrain (Tableau 2). Le principal risque est la dégradation de l'habitat favorable à l'espèce et la destruction directe d'individus. Pour cette raison, nous recommandons:

- Un contrôle de la fréquentation humaine (sentiers balisés, mises en défens) et une fermeture des milieux afin de préserver l'habitat de l'espèce.

- Une sensibilisation du public local pour respecter la flore et ses habitats tout en adoptant spontanément un comportement intentionné vis-à-vis de sa fragilité. - Une étude des aspects de reproduction et de colonisation des milieux, ainsi qu'une surveillance des effets causés par les incendies sur les taux de germination des graines et leur survie dans le sol ; car à ce jour, on manque de connaissances sur certains aspects écologiques de l'espèce.

- Selon nos observations sur le terrain, la colonisation par $O$. algeriense est limitée par la prolifération de la Cétoine noire à points blancs (Oxythyrea funesta Poda, 1761) (Fig. 4).

- II serait très important de faire une conservation Exsitu dans des pépinières expérimentales pour mieux déterminer les conditions idéales pour la germination des graines et pour le développement de cette espèce.
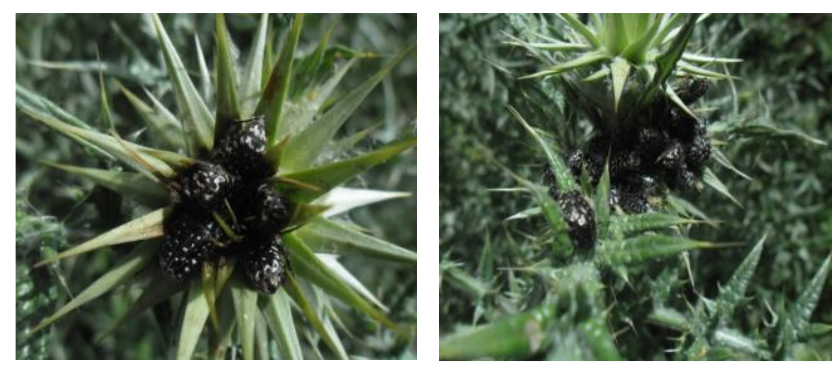

Figure 4. Attaque massive de la cétoine funeste sur les capitules d'Onopordum algeriense (Munby) Pomel.

Figure 4. Massive attack of the fatal Cetonia on the flower heads of Onopordum algeriense (Munby) Pomel.

\section{Statut de l'espèce par rapport aux critères de I'UICN:}

Onopordum algeriense est non seulement une espèce endémique mais une espèce très rare, car localisée dans un seul endroit près d'Alger et sur quelques mètres carrés.

Son statut actuel selon I'UICN est «gravement menacée » (UICN, 2018).

L'UICN a établi des critères en vue de définir le statut des espèces comme spécifié dans les tableaux qui suivent. On a soumis ainsi $O$. algeriense à ce filtre, il en ressort ce qui suit:

Si on adopte la catégorie $A$ (réduction de la taille de la population), les données actuelles ne nous permettent pas de l'utiliser (Tableau 3).

La catégorie $B$ relative à la répartition géographique montre que la zone d'occupation est $<10 \mathrm{~km}^{2}$ ce qui définit l'espèce comme en danger critique (Tableau 4).

Par ailleurs la catégorie $C$ (petite population en déclin) n'est pas constatée (Critères C1, C2) non satisfaits (Tableau 5). 
Tableau 2: Principaux phénomènes pouvant affecter l'abondance d'Onopordum algeriense (Munby) Pomel.

Table 2: Main phenomena that can affect abundance of Onopordum algeriense (Munby) Pomel.

\begin{tabular}{|c|c|c|c|}
\hline Causes & Phénomènes & $\begin{array}{l}\text { Sensibilité } \\
\text { (Période) }\end{array}$ & Risques (conséquences) \\
\hline Végétation & $\begin{array}{l}\text { Changement drastique de couvert } \\
\text { végétal } \\
\text { (Défrichement, plantations...) }\end{array}$ & Toute l'année & $\begin{array}{l}\text { Risque majeur d'extinction si modification } \\
\text { du milieu par plantation }\end{array}$ \\
\hline \multirow[t]{3}{*}{ Sol } & $\begin{array}{l}\text { Terrassements (remblais, } \\
\text { compactages) }\end{array}$ & Toute l'année & Risque de l'extinction de l'espèce \\
\hline & $\begin{array}{l}\text { Forte artificialisation des sols } \\
\text { (goudron, béton) }\end{array}$ & Toute l'année & Risque de l'extinction de l'espèce \\
\hline & $\begin{array}{l}\text { Ouvrage hydraulique linéaire, avec } \\
\text { rupture de continuités écologiques }\end{array}$ & Toute l'année & $\begin{array}{l}\text { Risque peu probable a priori, cependant } \\
\text { fragmentation suite à un gabionnage }\end{array}$ \\
\hline \multirow[t]{5}{*}{ Homme } & Rejets (déchets) & Toute l'année & Risque de dégradation du milieu \\
\hline & Urbanisation (bidonvilles) & Toute l'année & Risque de l'extinction de l'espèce \\
\hline & $\begin{array}{l}\text { Sur-fréquentations humaines } \\
\text { (piétinements, pique-niques, } \\
\text { déplacements en motos, } \\
\text { pâturages) }\end{array}$ & Toute l'année & $\begin{array}{l}\text { Risque de dégradation du milieu favorable } \\
\text { à l'espèce }\end{array}$ \\
\hline & Incendies & Saison estivale & $\begin{array}{l}\text { Risque de dégradation du milieu favorable } \\
\text { à l'espèce }\end{array}$ \\
\hline & Raréfaction des pollinisateurs & $?$ & Risque de l'extinction de l'espèce \\
\hline Climat & Changement climatique & $?$ & $\begin{array}{l}\text { Risque de dégradation du milieu favorable } \\
\text { à l'espèce }\end{array}$ \\
\hline Autre & $\begin{array}{l}\text { Attaques d'insectes (cétoine } \\
\text { funeste) }\end{array}$ & $\begin{array}{l}\text { Printemps- } \\
\text { floraison (sortie } \\
\text { des capitules) } \\
\end{array}$ & Extinction de l'espèce \\
\hline
\end{tabular}

Tableau 3: Critère A: Réduction de la taille de la population. Réduction (mesurée sur la plus longue des deux durées: 10ans ou 3 générations) sur la base d'un ou plusieurs des critères $A 1$ à $A 4$.

Table 3: Criterion A: Reduction of population size. Reduction (measured over the longer of the two durations: 10 years or 3 generations) on the basis of one or more of criteria $A 1$ to $A 4$

\begin{tabular}{|c|c|c|c|}
\hline \multicolumn{4}{|c|}{$\begin{array}{l}\text { A. Réduction de la taille de la population. Réduction (mesurée sur la plus longue des deux durées:10ans ou } \\
\text { 3génération) sur la base d'un ou plusieurs des critères A1 à A4 }\end{array}$} \\
\hline & $\begin{array}{l}\text { En danger } \\
\text { critique }\end{array}$ & En danger & Vulnérable \\
\hline $\mathrm{A} 1$ & $\geq 90 \%$ & $\geq 70 \%$ & $\geq 50 \%$ \\
\hline $\mathrm{A} 2, \mathrm{~A} 3 \& \mathrm{~A} 4$ & $\geq 80 \%$ & $\geq 50 \%$ & $\geq 30 \%$ \\
\hline $\begin{array}{l}\text { A1 Réduction de la population } \\
\text { constatée,estimée,déduite ou supposée,dans le } \\
\text { passé ,lorsque les causes de la réduction sont } \\
\text { clairement réversibles ET comprises ET ont } \\
\text { cessé } \\
\text { A2 Réduction de la population } \\
\text { constatée,estimée,déduite ou supposée,dans le } \\
\text { passé ,lorsque les causes de la réduction n'ont } \\
\text { peut-être pas cessé OU ne sont peut-être pas } \\
\text { comprises OU ne sont peut-être pas réversibles } \\
\text { A3 Réduction de la population prévue,déduiteou } \\
\text { supposée dans le futur(sur un maximum de } \\
100 a n s)[(a) n e \text { peut pas être utilisé pour A3] } \\
\text { A4 Réduction de la population constatée, } \\
\text { estimée, déduite, prévue ou supposée, sur une } \\
\text { période de temps devant inclure à la fois le } \\
\text { passé et l'avenir (sur un maximum de 100ans } \\
\text { dans le futur), lorsque les causes de la } \\
\text { réduction n'ont peut-être pas cessé OU ne sont } \\
\text { peut-être pas comprises OU ne sont peut-être } \\
\text { pas réversibles. }\end{array}$ & & $\begin{array}{l}\text { ant sur } \\
\text { éments }\end{array}$ & $\begin{array}{l}\text { (a) l'observation directe [excepté A3] } \\
\text { (b) un indice d'abondance adapté au } \\
\text { taxon } \\
\text { (c) la reduction de la zone d'occu- } \\
\text { pation(AOO), de la zone d'occu- } \\
\text { rrence(EOO)et/ou de la qualité de } \\
\text { l'habitat } \\
\text { (d) les niveaux d'exploitation réels ou } \\
\text { potentiels } \\
\text { (e) les effets de taxons introduits de } \\
\text { l'hybridation, d'agents pathogènes, } \\
\text { de substances polluantes, d'espèces } \\
\text { concurrentes ou parasites. }\end{array}$ \\
\hline
\end{tabular}


Tableau 4: critère B: Répartition géographique, qu'il s'agisse de B1 (zone d'occurrence) ET/OU B2

Table 4: Criterion B: Geographic Distribution, B1(extent of occurrence) AND / OR B2.

\section{B. Répartition géographique, qu'il s'agisse de B1 (zone d'occurrence) ET/OU B2 (zone d'occupation)}

\begin{tabular}{llll}
\hline & En danger critique & En danger & Vulnérable \\
B1.Zoned'occurrence ( EOO) & $<100 \mathrm{~km}^{2}$ & $<5000 \mathrm{~km}^{2}$ & $<20000 \mathrm{~km}^{2}$ \\
B2.Zoned'occupation ( $\mathrm{AOO})$ & $<10 \mathrm{~km}^{2}$ & $<500 \mathrm{~km}^{2}$ & $<2000 \mathrm{~km}^{2}$
\end{tabular}

ET au moins 2 des 3 conditions suivantes:

(a) Sévèrement fragmentée OU nombre de $=1 \quad \leq 5 \leq 10$ localités

(b) Déclin continu constaté, estimé, déduit ou prévu de l'un des éléments suivants:(i) zone d'occurrence, (ii)zone d'occupation, (iii) superficie, étendue

et /ou qualité de l'habitat,(iv) nombre de localités ou de sous-populations,(v)nombre d'individus matures

(c) Fluctuations extrêmes de l'un des éléments suivants: ( i ) zone d'occurrence,(ii) zone d'occupation, (iii) nombre de localités ou de sous-populations,

(iv) nombre d'individus matures

Tableau 5: Critère C: petite population en déclin.

Table 5: Criterion C: Small Population Declining

\section{Petite population et déclin}

\begin{tabular}{|c|c|c|c|}
\hline & En danger critique & En danger & Vulnérable \\
\hline Nombred'individus matures & $<250$ & $<2500$ & $<10000$ \\
\hline \multicolumn{4}{|l|}{ ET au moins un des sous-critères $\mathrm{C} 1$ ou $\mathrm{C} 2$} \\
\hline $\begin{array}{l}\text { C1. Un déclin continu constaté, estimé ou prévu } \\
\text { (sur un maximum de 100ans dans le futur)d'au } \\
\text { moins: }\end{array}$ & $\begin{array}{c}25 \% \text { en } 3 a n s \text { ou } 1 \text { génération } \\
\text { (sur la plus longue des deux } \\
\text { durées) }\end{array}$ & $\begin{array}{c}20 \% \text { en } 5 \text { ans } \\
\text { ou } 2 \\
\text { générations } \\
\text { (sur la plus } \\
\text { longue des } \\
\text { deux durées) }\end{array}$ & $\begin{array}{l}10 \% \text { en } 10 \text { ans ou } \\
\text { 3générations (sur } \\
\text { la plus longue des } \\
\text { deux durées) }\end{array}$ \\
\hline
\end{tabular}

C2. Un déclin continu constaté, estimé, prévu ou déduit ET au moins 1 des 3 conditions suivantes:

(i)Nombre d'individus matures dans chaque $\leq 50$

$\leq 250$

$\leq 1000$ sous -population:

(a) (ii) \% d'individus matures dans une souspopulation $=$

$90-100 \%$

$95-100 \%$

$100 \%$

(b)Fluctuations extrêmes du nombre d'individus matures

Tableau 6: Critère D: population très petite ou restreinte.

Table 6: Criterion D: Very Small or Restricted Population

\begin{tabular}{llll}
\hline D. Population très petite ou restreinte & & & \\
\hline & En danger critique & En danger & Vulnérable \\
D.Zoned'occurrence (EOO) & $<50$ & $<250$ & D1.<100 \\
$\begin{array}{l}\text { D2.pour la catégorie VU uniquement Zone } \\
\text { d'occupation restreinte ou nombre de localités }\end{array}$ & - & D2.en règle \\
limité et susceptibles d'être affectées à l'avenir & & générale: \\
par une menace vraisemblable pouvant très vite & & AOO<20KM² ou \\
conduire le taxon vers EX ou CR & & nombre de \\
\hline
\end{tabular}


Tableau 7: Critère E. Analyse quantitative.

Table 7: Criterion E: Quantitative analysis

\begin{tabular}{|c|c|c|c|}
\hline E. Analyse quantitative & & & \\
\hline $\begin{array}{l}\text { Indiquant que la probabilité d'extinction dans la } \\
\text { nature est: }\end{array}$ & $\begin{array}{l}\text { En danger critique } \\
\geq 50 \% \text { sur } 10 \text { ans ou } \\
\text { 3générations, sur la plus } \\
\text { longue des deux durées } \\
\text { (100ans max) }\end{array}$ & $\begin{array}{l}\text { En danger } \\
\geq 20 \% \text { sur } \\
20 \text { ans ou } \\
\text { 5générations, } \\
\text { sur la plus } \\
\text { longue des } \\
\text { deux durées } \\
\text { ( } 100 \text { ans max) }\end{array}$ & $\begin{array}{l}\text { Vulnérable } \\
\geq 10 \% \text { sur } 100 \text { ans }\end{array}$ \\
\hline
\end{tabular}

La catégorie $D$ (population très petite ou restreinte), même si elle définit un nombre d'individus matures $<50$, s'applique parfaitement pour le critère D2, O. algeriense est susceptible de disparaitre à l'avenir par l'effet de l'urbanisation. Ce qui définit l'espèce comme vulnérable (Tableau 6).

La catégorie $E$ (analyse quantitative) semble indiquer que, depuis la découverte de l'espèce par Munby, en 1855, la population d' $O$. algeriense est stable, mais cela ne présage en rien son avenir (Tableau 7).

Les documents consultés et les travaux réalisés montrent la vulnérabilité d'O.algeriense.

L'application des critères UICN confirme que l'espèce est en danger critique.

\section{Conclusion}

Ce travail préliminaire sur $O$. algeriense (Munby) Pomel, 165 ans après sa découverte, montre une population avec un effectif total de 1648 pieds,

Caractérisée par une hétérogénéité locale avec tendance à l'agrégation.

Onopordum algeriense est perturbée par la présence du sanglier, influant directement sur sa prolifération, et probablement aussi par la présence d'un coléoptère (la cétoine funeste) qui se nourrit de ses organes floraux, chose l'empêchant de coloniser d'autres milieux.

II semblerait que les conditions particulières de son habitat naturel ne sont donc pas indispensables à sa survie mais c'est l'absence de compétiteurs dans cet habitat qui représente un fort avantage.

Le maintien de cette espèce endémique dans ce milieu à l'abri des exploiteurs et compétiteurs consiste davantage en sa capacité à se maintenir dans une zone refuge de quelques mètres carrés.

Sous contrainte, l'espèce a fait un réel choix stratégique. Plutôt que d'étendre son aire de distribution, elle a choisi de rester rare, endémique, parce qu'elle n'a pas d'autres solutions pour survivre.

Selon les critères de l'UICN adoptées, l'espèce est en danger critique, son avenir semble incertain du fait de la pression de l'urbanisation.

\section{Remerciement}

Les auteurs remercient le personnel de la circonscription de Baïnem-Alger - (Direction Géné- rale des Forêts- Algérie) pour leur aide sur le terrain. Ce travail a été partiellement soutenu par la Fundação para a Ciência e Tecnologia (Portuguese Foundation for Science and Technology) à travers le projet stratégique UID/AMB/040085/2013 (CENSE).

\section{Références}

Baumel, A., Affre L., Véla E., Auda P., Torre F., Youssef S., Tatoni T. (2009). Ecological magnitude and fine scale dynamics of the Mediterranean narrow endemic therophyte, Arenaria provincialis (Caryophyllaceae). Acta Botanica Gallica 156: 259-272.

Faurel, L., (1959). Plantes rares et menacées d'Algérie. C. R. de la réunion technique de I'UICN, $5,140-155$. Athènes.

Fortin, M. J., Dale, M. R. T. (2005). Spatial analysis: $A$ guide for ecologists. Cambridge Univ. Press, $365 \mathrm{p}$.

ISL-BRGM (2006). Etude de réduction de la vulnérabilité du massif de Bouzaréah aux catastrophes naturelles. Rapport de tâche 2, volume 2, R3114, 6-42.

IUCN (2018). Guide pratique pour la réalisation de Listes rouges régionales des espèces menacées Méthodologie de l'UICN \& démarche d'élaboration. Seconde édition. Paris, France.

Médail, F., Myers, N. (2004). Mediterranean Basin, in: R.A. Mittermeier, P. Robles Gil, M. Hoffmann, J. Pilgrim, T. Brooks, C.G. Mitter-meier, J. Lamoreux, G.A.B. da Fonseca (Eds.), Hotspots revisi-ted: Earth's Biologically Richest and Most Endangered Terres-trial Ecoregions, CEMEX, Conservation International \& Agru-pación Sierra Madre, Monterrey, Washington, Mexico, 144-147

Petit, R. J., Aguinagalde, I., Beaulieu de, J-L., Bittkau, C., Brewer, S., Cheddadi, R., Ennos, R., Fineschi, S., Grivet, D., Lascoux, M., Mohanty, A., MüllerStarck ,G., Demesure-Musch, B., Palmé, A., Martin, J. P., Rendell, S. \& Vendramin, G. G. (2003). Glacial refugia: hot spots but not melting pots of genetic diversity, Science, 300, 1563-1565.

Quézel, P. \& Médail, F. (2003). Écologie et biogéographie des forêts du Bassin méditerranéen, Elsevier, Paris. 573 pp.

Véla, E., Auda, P., Léger, J. F., Gonçalves, V., Baumel, A. (2008). Exemple d'une nouvelle éva- 
luation du statut de menace suivant les critères de I'UICN version 3.1.: le cas de l'endémique provençale Arenaria provincialis Chater \& Halliday (= Gouffeia arenarioides DC., Caryophyllaceae). Acta Botanica Gallica 155: 547-562.

Vela, E. \& Benhouhou, S. (2007). Évaluation d'un nouveau point chaud de biodiversité végétale dans le Bassin méditerranéen (Afrique du Nord). Comptes-Rendus Biologies, 330, 589-605.
Youssef, S., Baumel, A., Véla, E., Juin, M., Dumas, E., Affre, L., Tatoni, T. (2011). Factors underlying the narrow distribution of the Mediterranean annual plant Arenaria provincialis (Caryophyllaceae). Folia Geobotanica published online 25 Mai 2011.

Zuur, A. F., leno, E.N., Smith, G.M., (2007). Analysing ecological data. Springer, New York. 672p 